\title{
Spectral fissioning in phonemic transformations
}

\author{
MAGDALENE H. CHALIKIA and RICHARD M. WARREN \\ University of Wisconsin-Milwaukee, Milwaukee, Wisconsin
}

\begin{abstract}
Listeners presented with a repeated sequence of brief (30- to 100-msec) steady-state vowels hear phonemic transformations-they cannot identify the vowels, but they perceive two simultaneous utterances that differ in both phonemic content and timbre (Warren, Bashford, \& Gardner, 1990). These utterances consist of either English words or syllables that occur in English words. In the present study, we attempted to determine whether the two percepts represent alternative interpretations of the same formant structures, or whether different portions of the vowels are used for each verbal organization. It was found that separate spectral regions are employed for each verbal form: Components below $1500 \mathrm{~Hz}$ were generally used for one form, and components above $1500 \mathrm{~Hz}$ for the other. Hypotheses are offered concerning the processes responsible for the verbal organization of the vowel sequences and for the splitting into two spectrally limited forms. It appears that the tendency to organize spectral regions separately competes with, and can overcome, the tendency to integrate the different spectral components of speech into a single auditory image. A contralateral induction paradigm was used in a procedure designed to quantitatively evaluate these opposing forces of spectral fission and fusion.
\end{abstract}

When listeners are presented with recycled sequences of brief vowels, phonemic transformations occur, and the listeners usually hear two simultaneous voices that have different timbres, each voice pronouncing different syllables or words (Chalikia \& Warren, 1991; Warren, Bashford, \& Gardner, 1990). In the present investigation, we examine the spectral characteristics and properties of these concurrent verbal forms, and we present hypotheses that relate the experimental findings to mechanisms normally employed for verbal organization.

In a number of studies, recycled sequences of three or four vowels have been used to study the minimum item duration required for the identification of the temporal order of phonetic segments (Cole \& Scott, 1973; Cullinan, Erdos, Schaefer, \& Tekieli, 1977; Dorman, Cutting, \& Raphael, 1975; Thomas, Cetti, \& Chase, 1971; Thomas, Hill, Carroll, \& Garcia, 1970; Warren, Obusek, Farmer, \& Warren, 1969; Warren \& Warren, 1970). It was found that naming of vowels in their proper order was easy at $200 \mathrm{msec} / \mathrm{item}$, but was not possible below $100 \mathrm{msec} / \mathrm{item}$. Brief silent intervals between steady-state components or the introduction of acoustic transitions resembling those linking adjacent phonemes in speech facilitated the task, but order identification could not be accomplished under

This research was supported by grants to the second author from the National Institutes of Health (DC00208) and the Air Force Office of Scientific Research (88-0320). The results were presented at the 120th meeting of the Acoustical Society of America. The valuable contributions of Jim Bashford, Brad Brubaker, and Keri Riener are gratefully acknowledged, as are the comments of Eric Healy and Makio Kashino. Correspondence may be sent to either M. H. Chalikia, Department of Psychology, Moorhead State University, Moorhead, MN 56560, or R. M. Warren, Department of Psychology, University of WisconsinMilwaukee, Milwaukee, WI 53201.

-Accepted by previous editor, Charles W. Eriksen any of the conditions employed when item durations were below $100 \mathrm{msec}$. This 100 -msec limit for temporal resolution seems curious if it is considered that speech comprehension requires recognition of phonemes in their proper order since vowels and other phonemes in speech can have considerably shorter durations-indeed the average duration of phonemes in ordinary conversation can approximate $80 \mathrm{msec} /$ phoneme (Efron, 1963), and artificially "compressed speech" preserves some intelligibility when the average duration of phonemes is as brief as $30 \mathrm{msec}$ (Foulke \& Sticht, 1969).

There is considerable evidence that listeners can distinguish between different arrangements of brief nonverbal sounds such as tones, hisses, and buzzes even when they cannot identify their order (for a review of this topic, see Warren, 1993). Therefore, it seems plausible that listeners can discriminate between (and recognize) permuted orders of brief steady-state vowels below the threshold for order identification. On the basis of this reasoning, Warren et al. (1990) employed different arrangements of recycled sequences of three steady-state vowels $/ / \AA æ i \Lambda æ i \Lambda . . /$ and

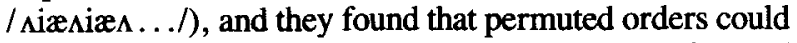
be distinguished with ease at all durations down to $10 \mathrm{msec} /$ vowel (corresponding to a single glottal pulse of each phoneme). At the briefest durations-from $10 \mathrm{msec} / \mathrm{item}$ to $30 \mathrm{msec} / \mathrm{item}$ - the concatenated vowels did not sound like speech sounds, and the sequences were distinguished by differences in timbre. For item durations ranging from $100 \mathrm{msec} /$ item down to $30 \mathrm{msec} / \mathrm{item}$, a compelling illusion was observed: The sequences were transformed perceptually into syllables and words, with the different arrangements corresponding to different verbal organizations. This phonemic transformation of components occurred even though vowels throughout this range of durations could be identified readily when presented in 
isolation. It appears that the phonemes in such sequences undergo an obligatory integration into verbal organizations from which listeners can identify only the illusory forms corresponding to the particular arrangements, and not the series of vowels actually present. Phonemic transformations have another characteristic of interest-they usually involve two simuitaneous verbal forms that have distinctly different timbres. Occasionally, only one verbal form can be heard along with a nonverbal residue.

In addition to employing recycled sequences of three vowels (for which there are two possible orders), Warren et al. (1990) also employed recycled sequences of 10 different $40-\mathrm{msec}$ vowels (for which there are over 300,000 possible orders), and found that verbal mediation permitted listeners to distinguish readily between pairs consisting of randomly determined arrangements. Again, perceptual splitting was observed so that two simultaneous forms could usually be heard for each sequence. Even with minimal differences in the arrangement of the 10 vowels (permuting the order of two contiguous items), listeners were still able to hear verbal forms that differed for the two arrangements.

Amano, Kashino, and Warren (1990) extended the study of phonemic transformations and their characteristics to a language other than English. They used recycled sequences of the five Japanese vowels $(60-\mathrm{msec}$ item durations) and found that the listeners, who were native speakers of Japanese, heard moras or syllables in their native language. As with English speaking subjects hearing English vowels, the Japanese listeners reported hearing concurrent verbal forms.

Chalikia and Warren (1991) determined the correspondence of each of the phonemes within an illusory English syllable or word to the steady-state vowel actually present at that time. The mapping technique used had been employed previously for determining the perceptual boundaries of phonemes while subjects listened to assortments of syllables, words, and sentences (Warren, 1971; Warren \& Sherman, 1974). When (as was usual) a vowel sequence was heard as two simultaneous forms, it was found that the more prominent or primary form usually had a greater phonetic complexity. Also, the stimulus vowels corresponding to the initial phonemes of the two forms were usually different. It was noted that the linguistic organizations reported for both simultaneous forms followed the rules governing the sequencing of phonemes in English. A further restriction was observed that had implications for theories dealing with the initial stages in the organization of speech. It was found that although nonlexical verbal items were reported frequently $(50 \%$ or more of the responses in some of our experiments), these items consisted of syllables found within English words-a form such as flime would not be reported, even though it conforms to the phonotactic rules of English. Thus, it appears that listeners may be able to access directly a syllabary that consists of only those syllables that actually occur in English.
The perceptual fissioning of steady-state vowel sequences into two voices is rather curious; splitting does not occur spontaneously with a steady-state vowel heard by itself, or with the phonetic segments that form normal utterances. The present study was undertaken to explore the bases for this fission, and its relation to mechanisms governing the perceptual organization of speech. The difference in the timbre of the simultaneous voices suggested that it might be possible, through filtering, to isolate separate spectral regions corresponding to each verbal form. This possibility was examined in Experiment 1: Listeners were instructed to adjust the upper and lower limits of a bandpass filter in order to isolate each verbal form from the other. They were successful in locating separate spectral regions corresponding to the individual forms.

Usually, of course, all spectral regions of vowels are integrated and heard as single auditory images, whether they are presented in isolation or as components of normal utterances. However, when a sequence of steady-state vowels is presented to a listener, processes associated with verbal organization appear to overcome this spectral cohesion, and separate images are heard for low-frequency and for high-frequency components. Experiment 2 was designed to measure the relative magnitudes of the opposing forces of spectral cohesion and spectral fission in vowel sequences by pitting one force against the other in a contralateral induction paradigm.

\section{EXPERIMENT 1}

This experiment was designed to select a set of recycled vowel sequences for each listener that would produce two salient verbal forms, and to determine whether it was possible, through bandpass filtering, to isolate the spectral components contributing to each form.

\section{Method}

Subjects. Eight listeners with normal hearing were employed. They had all been participants in other studies carried out in the laboratory.

Stimuli. Each vowel sequence was made up of ten $60-\mathrm{msec}$ vowels $(/ \mathrm{J} /$ as in hawd, /I/ as in hid, / / / as in hod, /i/ as in heed, /a/ as in heard, / / / as in head, / $/ \mathrm{s} /$ as in hood, /æ/ as in had, /u/ as in hoot, and $/ \Lambda /$ as in hud). Figure 1 shows the spectrograms of these vowels. For synthesis of each vowel, a digitizer (operating at a sampling rate of $22 \mathrm{kHz}$ with 8-bit resolution) was used to excise single 10 -msec glottal pulses from a male speaker's sustained productions of each vowel $(100-\mathrm{Hz}$ voicing frequency was produced while the production was matched to a $100-\mathrm{Hz}$ tone delivered through headphones). Each excised glottal pulse started and ended at zero crossing, in order to minimize acoustic transients. The individual digitized glottal pulses were each iterated six times to produce 60 msec segments. Because of differences in spectral composition, vowels at equal $\mathrm{dBA}$ did not sound equally loud. To compensate for this difference, the amplitude envelopes of the segments were each adjusted to the minimal level required for identification of the vowel when it was presented alone in the presence of a 70-dBA pink noise, as judged by one of the experimenters. The resulting intensity difference spanned by the vowels was $8 \mathrm{dBA}$. The 10 vowel segments were sampled randomly without replacement and con- 


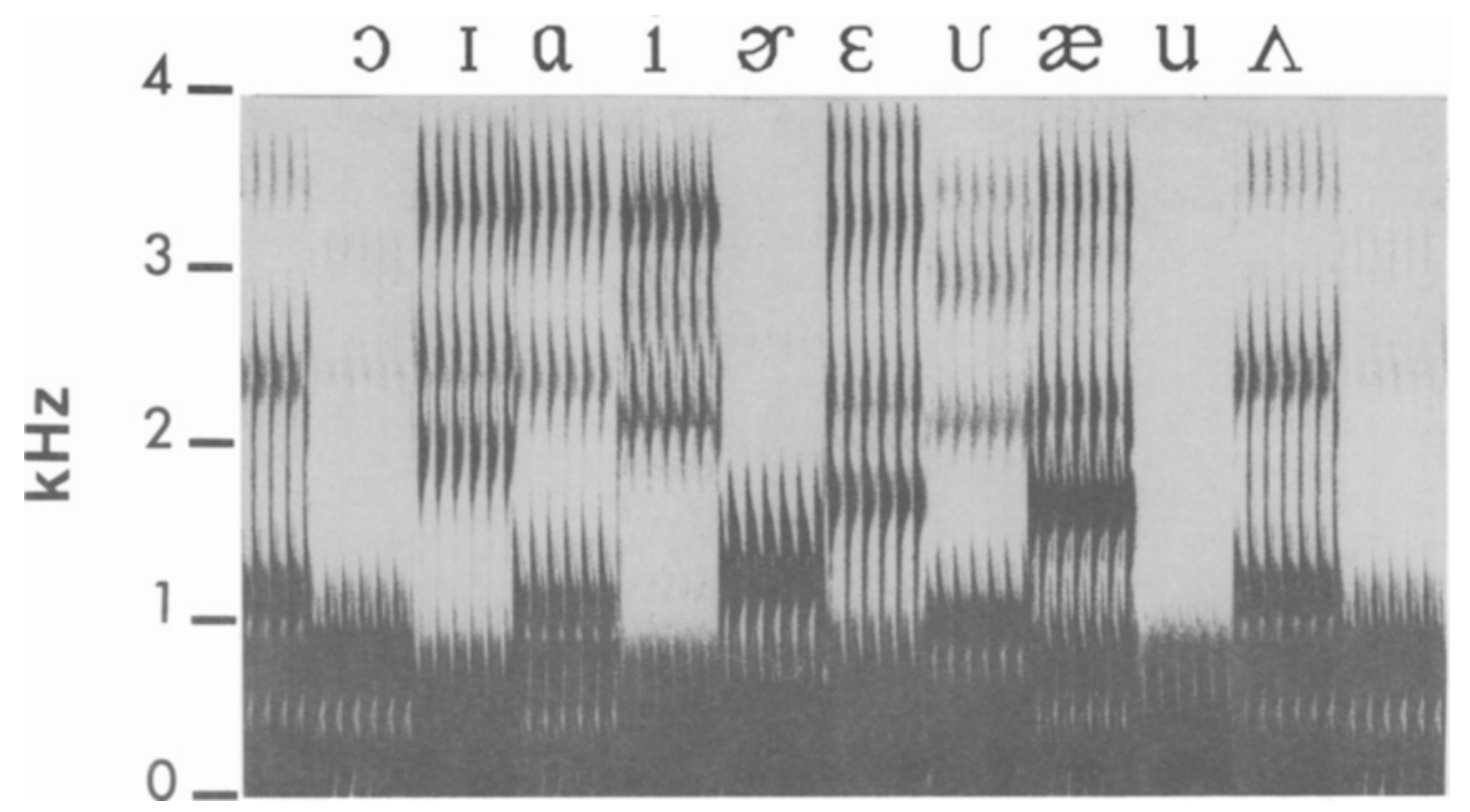

Figure 1. Spectrograms of the 10 vowels used (those in hawd, hid, hod, heed, heard, head, hood, had, who'd, and hud).

catenated in digital form to create 78 different 10 -item sequences (out of a total of factorial 9 , or 362,880 , possible orderings). One of the experimenters listened to these 78 sequences and selected as potential stimuli 24 that readily gave rise to two simultaneous verbal forms. The analog playback of the recycling sequences was recorded on an Otari Model MX5050 8-track recorder, with 3 sequences recorded on each track. During the experiment, the output of the recorder was amplified by a Ramsa Model WR-8118 audio mixer and bandpass filtered from 50 to $8000 \mathrm{~Hz}$ with slopes of $48 \mathrm{~dB}$ /octave (Wavetek Model 852 Filter).

Procedure. The listeners were tested individually in an audiometric room with the stimuli delivered diotically through Sennheiser HD230 headphones at an overall level of $55 \mathrm{dBA}$ (measured by a Brüel and Kjaer Type 2230 sound-level meter with slow meter response). The experimenter, seated in the room with the listener, operated the Otari recorder (located outside the chamber) with a remote, preset search-to-cue device. Switches on the audio mixer located inside the chamber permitted delivery of the output from the desired tracks of the recorder.

The experiment consisted of three sessions. The first lasted $1 \mathrm{~h}$. The subsequent two sessions lasted about half an hour each. During the first session, all listeners heard the 24 sequences in the same order. They were instructed to write down on an answer sheet any words that they heard (with the more prominent form first when they heard two) and to rate the clarity of each form on a 5-point scale. (As discussed earlier, a vowel sequence is generally heard as two simultaneous verbal forms, one often being more prominent than the other.) After this session, the experimenter inspected all the answer sheets and selected, for each listener, five sequences for which both forms had received high clarity ratings. During the second session, each listener verified that the pairs of forms reported previously for the individual sequences could be heard again. They then attempted to isolate each member of the pair of simultaneous forms from the other by using the low-pass and high-pass frequency control knobs of the filter (the calibrated dials were hidden from view). Finally, they determined the narrowest range of frequencies required for the perception of each of the forms by ad- justing the low-pass and high-pass settings of the filter. After each adjustment was completed, the experimenter recorded the filter settings. During the third session, the listeners were presented with the filtered bands that had been selected earlier, in order to verify that only the one form reported previously could be heard for each filter setting selected for each of the five arrangements of vowels.

\section{Results}

During the first session, the perceptual organization of the recycling vowel sequences into syllables and words proved effortless. There were individual differences in the sequences producing two forms rated as having matching high clarity, but the experimenter was able to select five of the sequences for each listener that had produced simultaneous forms with matching high clarity. During the second session, all listeners ascertained that the simultaneous forms reported originally could be heard again. Six of the 8 subjects were able (through filtering) to isolate their two forms into distinct spectral regions for each of the five sequences used. The other 2 listeners reported that there was a frequency overlap of the two regions for at least one of their five sequences, and, since they could not isolate each of their forms, they were dropped from the study. During the third session, each listener confirmed that the form reported previously for a particular frequency band could be heard again. After this session, 1 of the 6 remaining listeners reported being unable to continue participation. Consequently, the reported results are based on the participation of 5 listeners. Table 1 shows the vowel sequences employed for each listener, along with the two forms heard for each sequence and the 5-point clarity rating for each form. It can be seen in Table 1 that, although the exact location and width of the selected spec- 
Table 1

Spectral Bands and Clarity Ratings (on a 5-Point Scale) Selected by Listeners for Pairs of Simultaneous Verbal Forms Heard With Repeated Sequences of Steady-State Vowels

\begin{tabular}{|c|c|c|c|c|c|c|c|}
\hline Subject & Sequences & Form A & Band (Hz) & Clarity & Form B & Band (Hz) & Clarity \\
\hline \multirow[t]{5}{*}{1} & 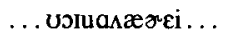 & he did & $300-600$ & 5 & par-el-kaw & $1000-2500$ & 5 \\
\hline & . . . sluiaruæor . . . & awe-path-awe & $400-1200$ & 5 & *tha-kate-vee & $1400-3000$ & 5 \\
\hline & . . EInJuaviæræ . . . & pupa & $500-1100$ & 5 & belong-awe & $1700-3500$ & 5 \\
\hline & 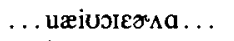 & awe-paw-peer-uh & $500-1200$ & 5 & he did & $1800-3000$ & 5 \\
\hline & 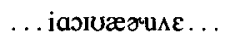 & key-paul-deh & $500-1100$ & 5 & eye-it-eat-ah & $1500-2400$ & 5 \\
\hline \multirow[t]{5}{*}{2} & 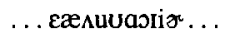 & helper & $300-1500$ & 5 & stay back & $1700-5000$ & 5 \\
\hline & . . ucuarasaniı . . & cloth & $100-1500$ & 5 & be in & $1500-4000$ & 5 \\
\hline & ... anæuaseliu... & follow & $300-1400$ & 5 & packer & $1500-4000$ & 5 \\
\hline & 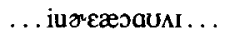 & foreman & $200-1200$ & 5 & back pay & $1500-4000$ & 5 \\
\hline & . . . Iiઝuruæeas . . . & holder & $300-1400$ & 5 & paper & $1500-3500$ & 4 \\
\hline \multirow[t]{5}{*}{3} & 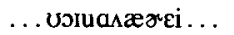 & bah-rickle & $800-1000$ & 3 & eat it & $1700-5000$ & 3 \\
\hline & 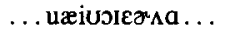 & ak-oh-furlah & $500-1200$ & 3 & detail & $1800-2100$ & 3 \\
\hline & 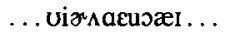 & brupple & $700-900$ & 3 & a tidy & $1400-2500$ & 3 \\
\hline & . . . Iijuruacan . . . & glottal & $600-1200$ & 4 & ah-duh-pur & $1500-2000$ & 4 \\
\hline & . . Iæa^əueuji. . . & potter ripple & $800-1000$ & 3 & bee-akerman & $1500-1900$ & 3 \\
\hline \multirow[t]{5}{*}{4} & 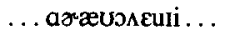 & au revoir & $100-1300$ & 4 & ah-day-ee & $2300-3900$ & 4 \\
\hline & . . . sıæißuuarea . . . & eh-hee & $300-1100$ & 4 & low-neck & $2600-3500$ & 4 \\
\hline & . . ^عiUariasua . . . & ah-er-uh & $300-1500$ & 5 & ah-ee-eye & $1500-5000$ & 5 \\
\hline & 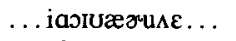 & ba-bough-dover & $100-1200$ & 5 & uh-eye-eh & $1900-3900$ & 5 \\
\hline & . . . мiæsueraru. . . & uh-or-luh & $690-1000$ & 4 & hack-uh-dee & $1900-4000$ & 5 \\
\hline \multirow[t]{5}{*}{5} & . . عænưasiz . . . & amalmer & $100-1200$ & 4 & bree-ah & $1100-3000$ & 5 \\
\hline & 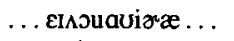 & balbulper & $100-1400$ & 5 & he paid & $1500-3500$ & 5 \\
\hline & . . u uæiuगır $\Lambda$ a . . . & lopla & $100-1000$ & 4 & eat it & $900-3500$ & 5 \\
\hline & 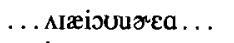 & uh-ah-hee-oo & $100-1100$ & 4 & eddie & $1400-3500$ & 5 \\
\hline & . . iasıææи & ma-malmet & $100-1000$ & 5 & eye-eh & $1400-3400$ & 5 \\
\hline
\end{tabular}

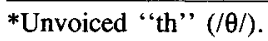

tral bands were different, usually one verbal form corresponded to a spectral region below roughly $1500 \mathrm{~Hz}$ and the other to a spectral region above that value.

Normally, of course, component frequencies of speech are integrated into a single auditory image. It appears that the tendency to split the vowel sequences into two separate images can overcome this tendency for the spectral integration of speech. In Experiment 2, we examined and measured the relative strengths of the competing forces of fission and fusion by using a contralateral induction paradigm to extract individual verbal forms from broadband vowel sequences.

\section{EXPERIMENT 2}

The contralateral induction paradigm is related to observations reported earlier. Egan (1948) found that when speech is delivered to one ear and noise to the other, the monaural speech seems to be displaced toward the side receiving the noise. Thurlow and Elfner (1959) found that a monaural tone could be "pulled in" by a contralateral tone that had a different frequency. Similar pulling effects were observed by Butler and Naunton $(1962,1964)$ when stimulating one ear with a signal delivered through a headphone while other sounds were delivered by a movable loudspeaker. However, the extent of this displacement toward the midline is unstable and difficult to measure because of the problem of lateralization drift. As one listens to a monaural sound for an extended period, its apparent position drifts toward the midsagittal plane (Bartlett \& Mark, 1922; Flugel, 1920).
Warren and Bashford (1976) reasoned that by regularly reversing the sides of the monaural target signal and the contralateral sound responsible for inducing delateralization, they might be able to obtain a stable measure of the pulling effect. This approach proved successful. Contralateral induction occurred, and the target signal (either a tone or speech) appeared to be completely stationary at a diffuse position centered on the medial plane, while the noise that served as the inducing sound was heard to move from side to side. The change from lateral movement of the target signal to a stationary image occurred quite abruptly when the intensity of the inducing sound reached a threshold value, making possible quantitative measures of the minimal level required for delateralization. It was found that contralateral induction represents a highly selective frequency-sensitive mechanism capable of delateralizing the monaural signal when it could be present as a masked component at the contralateral ear. The following rule was suggested for the contralateral induction of both speech and nonverbal signals: "If the peripheral neural units stimulated on the side receiving the louder sound include those corresponding to an ongoing contralateral signal, the fainter signal is released from lateralization and moves to a diffuse position located symmetrically about the median plane" (Warren \& Bashford, 1976, p. 384).

It has been reported that contralateral noise facilitates recognition of patterns disrupted by having alternate segments delivered to opposite ears. Cherry and Taylor (1954) reported that periodic switching of monaural speech from one side to the other decreased intelligibil- 
ity, and Schubert and Parker (1956) found that addition of broadband noise to the ear opposite to the one receiving the voice improved intelligibility. A similar facilitation of signal recognition was described by Deutsch (1979), who reported that recognition of melodic patterns delivered alternately to opposite ears was enhanced by the simultaneous presence of a "drone" signal at the contralateral ear. She noted that contralateral induction could enhance integration of the signal fragments delivered to opposite ears by moving the lateralized fragments to a common central locus.

Steiger and Bregman (1982) used contralateral induction to tease apart rather than to combine lateralized auditory images. They were interested in measuring the tendency of a sinusoidal target tone to interact with a sinusoidal "captor" tone. Their procedure involved alternation of the target tone delivered to one ear with the captor tone (which could vary in frequency) delivered to the same ear. In addition, a contralateral noiseband having the same center frequency as that of the target tone was presented along with the target (but was switched off when the captor was present). Steiger and Bregman reported that the tendency for contralateral induction produced by the noise to delateralize the target tone was opposed by the conflicting tendency for the target tone to remain lateralized due to "streaming" with the captor tone. By changing the captor frequency to diminish the tendency to stream, they could allow contralateral induction to dominate and to move the tone toward the medial plane. Thus, they used contralateral induction as a measure of the force binding elements of the target and captor tones into a single lateralized image.

In Experiment 2, we also employed contralateral induction to measure a force binding different elements together into a single auditory image. However, in our paradigm, we used the delateralization produced by contralateral induction to compete with and serve as a measure of the tendency of different spectral components of speech sounds to form integrated auditory images.

\section{Method}

Subjects. The 5 listeners who completed Experiment 1 participated in this study.

Stimuli. In the formal portion of this experiment, the seven broadband target signals described below were presented monaurally, along with a variety of contralateral narrowband sounds. These narrowband sounds were employed to extract and delateralize corresponding spectral regions of the broadband signals. The target signals were the five repeated vowel sequences selected for each listener in Experiment 1, and the repeated utterances "blandit" and "academic," with durations of $724 \mathrm{msec}$ and $737 \mathrm{msec}$, respectively. Repetitions of both the nonlexical utterance and the word were derived from single statements by the speaker responsible for the steady-state vowels. Recycling of utterances was accomplished by iterating the digital file corresponding to the utterance. Frequencies below $50 \mathrm{~Hz}$ and above $8000 \mathrm{~Hz}$ were remaved as in Experiment 1 for the vowel sequences, and the two utterances were then recorded on different tracks of the tape containing the vowel sequences.

On playback, the recordings of the vowel sequences and utterances were delivered to a Ramsa Model WR-8118 audio mixer and split into two parts. One part served as the monaural broadband $(50-8000 \mathrm{~Hz})$ target signal. The other part served as a narrowband contralateral inducer after the broadband signal was bandpass filtered with a Wavetek (Model 852) filter with slopes of $48 \mathrm{~dB} /$ octave. The cut-off frequencies for this bandpass filtering of the vowel sequences were adjusted by the experimenter for each listener, so that they corresponded to the settings for the individual forms heard with each of the five sequences as determined in Experiment 1 and shown in Table 1. A condition was also employed in which the monaural bandpassed speech serving as the inducer was replaced by bandpassed pink noise filtered in the same manner. When the target consisted of the utterances "blandit" or "academic," the monaural broadband signal was presented along with a narrowband contralateral inducer (derived from either the monaural broadband utterance or noise) that had a frequency range set to the average of all of the high bands or all of the low bands used with the vowel sequences for that listener.

Coulbourn electronic switches (Model S84-04) reversed the sides of the monaural inputs (the broadband signal and the contralateral inducer) at 0.7 -sec intervals as shown in Figure 2. Switching was performed with a linear rise/fall time of $10 \mathrm{msec}$ to eliminate audible spectral artifacts associated with switching. A mixer (Ramsa Model WR-133) was used to amplify the signals further. The level of the inducing sound (speech or pink noise) was controlled by means of a passive attenuator (Shallco, Type T693) calibrated in 1-dB steps. The pink noise was generated by a General Radio Company Random Noise Generator (Model 1382).

Procedure. In the main part of this experiment, the listeners were tested individually in an audiometric booth. The broadband speech signal was delivered to one ear at $55 \mathrm{dBA}$ through Sennheiser HD230 headphones, and the narrowband inducer was delivered to the opposite ear at a level controlled by the listener. The sides receiving the broadband target signal and the narrowband inducer were reversed each $0.7 \mathrm{sec}$, as is shown in Figure 2 . The experimenter operated the Otari recorder (located outside the booth) with a remote preset search-to-cue device. Switches on the audio mixer (WR8118) located inside the booth permitted delivery of the output from the desired tracks of the recorder, as well as the selection of the particular inducer delivered to the ear opposite to the one receiving the target signal.

There were five sessions, each lasting approximately $30 \mathrm{~min}$. During each session, the monaural broadband voice (the five vowel sequences appropriate for each listener as well as the repeated utterances "academic" and "blandit") were presented three times each in a randomized order. The sessions differed only in the nature of the contralateral inducer. In Session A, the inducer was a $50-8000 \mathrm{~Hz}$ broadband pink noise. In Sessions B and C, the inducer was a frequency-limited band obtained by filtering the contralateral vowel sequence or natural utterance. For the vowel sequences, the center frequencies and widths of the inducer bands were those determined in Experiment 1 (and shown in Table 1) for the verbal form corresponding to the lower frequency range (LoVoice) and for the verbal form corresponding to the higher frequency range (HiVoice), respectively. In Session D, the inducers

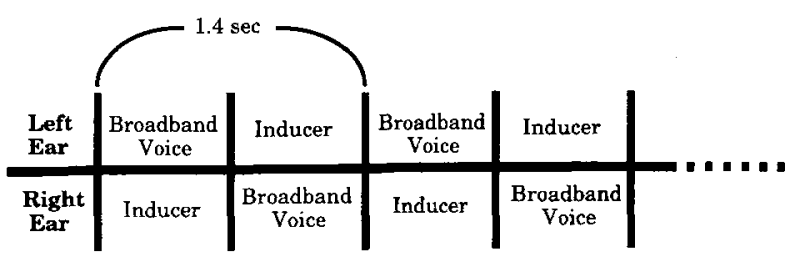

Figure 2. Alternating pattern of dichotic stimulation used for producing contralateral induction. 
were bands of pink noise (LoNoise) that matched the frequency range of LoVoice in Session B. In Session E, the inducing sounds were bands of pink noise (HiNoise) that matched the range of HiVoice in Session C. For the two utterances ("blandit" and "academic"), the low and the high inducers in Sessions B through E were set to be the average of all the low or all the high bands for each particular listener.

During each session, the listeners were told to attend either to both voices (when the inducer was broadband noise) or to only one of the voices (either the low or the high voice, depending on the particular band-limited inducer). The initial level of the inducing sound was set at a level near threshold and hence was well below that required for contralateral induction, and the subjects were instructed to use the dial of the attenuator to adjust the level of the inducer until they could just hear the appropriate verbal form as stationary and diffusely localized about the midsagittal plane (if this were not possible, they were to inform the experimenter). The difference between the level of the narrowband inducer and the level of the corresponding spectral region of the broadband speech signal required to produce spectral splitting was taken as a measure of the signal's degree of spectral cohesion, with higher inducer levels indicating greater cohesion.

\section{Results and Discussion}

In Session A, it was possible to delateralize completely each of the broadband monaural target signals, whether vowel sequences or utterances, when the broadband contralateral inducing noise was, on the average, about $20 \mathrm{~dB}$ higher in level. In keeping with the report of Warren and Bashford (1976), when the delateralization threshold was reached, the louder inducing noise moved the entire monaural signal to the center, where it was heard as stationary while the noise was heard as switching sides.

While it was possible to delateralize corresponding spectral regions of the monaural broadband vowel sequences with either contralateral band-limited portions of the sequences (Sessions B and C) or narrowband noise (Sessions $\mathrm{D}$ and $\mathrm{E}$ ), it was not possible to extract narrowband spectral regions when the broadband target signal was an actual utterance (either "academic" or "blandit") under any of the conditions employed, indicating the high degree of cohesion across spectral regions for the auditory image produced by normal speech. The analysis of spectral splitting, therefore, was based solely on the results obtained with the vowel sequences.

Figure 3 summarizes the pooled data obtained with vowel sequences for the group of 5 subjects ( 75 judgments were made for each condition: 3 judgments with 5 sequences for 5 subjects). The contralateral band of noise spanning the same frequency range as that of one of the verbal forms was able to extract that form from the shifting lateral positions occupied by the auditory images of the broadband vowel sequence and to move the particular verbal form to a stationary position centered on the medial plane. Contralateral induction of individual forms took place for all listeners with each type of inducer (LoNoise, HiNoise, LoVoice, and HiVoice). As can be seen in Figure 3, the level difference required for delateralization by narrowband voice is appreciably lower than the corresponding level difference for narrowband noise (Wilcoxon $z=2.02, p<.04$ ). ${ }^{1}$

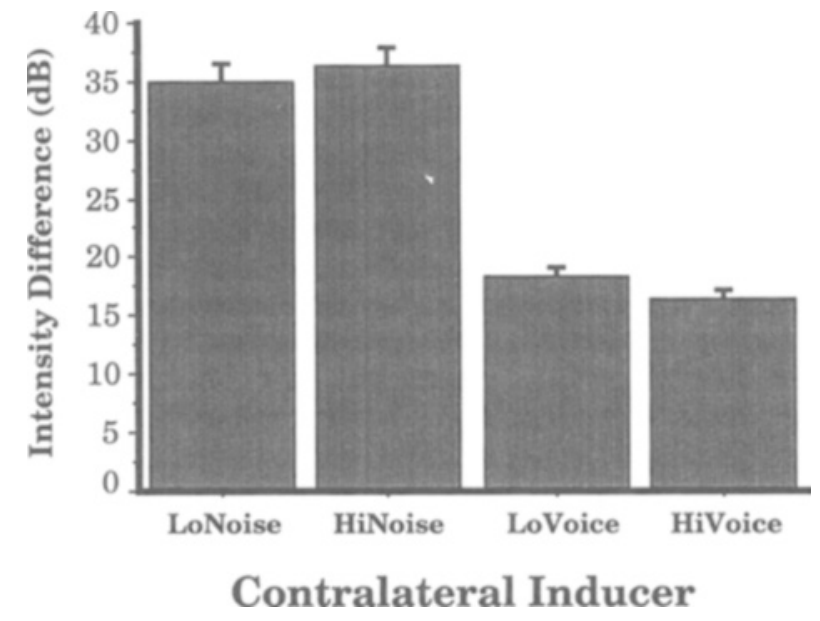

Figure 3. Interaural level differences required for delateralization of high-frequency and low-frequency verbal organizations by inducers with matching frequency ranges. Group means are shown along with their confidence intervals. For further description, see text.

It is of interest that although narrowband inducers consisting of either voice or noise could produce delateralization of corresponding spectral regions of the vowel sequences, neither of these could produce spectral splitting with the utterances "blandit" and "academic." Also, the informal presentation of extended statements of monaural single vowels in isolation demonstrated that neither narrowband noise nor a narrowband portion of the vowel could produce delateralization at any of the levels employed with vowel sequences. Since isolated vowels as well as the phonemes present in normal utterances resist spectral extraction (signifying a high cohesion across spectral regions), it appears that a vowel's presence in a sequence with other steady-state vowels markedly reduces these cohesive spectral forces by introducing competing band-limited processes that result in separate verbal organizations for different spectral regions. ${ }^{2}$

\section{GENERAL DISCUSSION AND CONCLUSIONS}

It has been reported in earlier studies that sequences of brief steady-state vowels are perceptually transformed into syllables and words, with individual sequences typically perceived as simultaneous verbal forms having different timbres by speakers of English (Chalikia \& Warren, 1991; Riener and Warren, 1990; Warren et al., 1990) and by speakers of Japanese (Amano et al., 1990).

In considering possible bases for the organization of vowel sequences into syllables and words, Warren et al. (1990) started with the concept that there is a strong tendency to organize a series of speech sounds linguistically, and that this organization is accomplished by matching the auditory input to linguistic templates employed for the identification of syllables and words. These templates have 
fuzzy acceptance boundaries and can be activated by tokens produced by the voices of children, women, and men, as well as by voices having different accents and idiosyncratic characteristics. Even though the limits for matching are broad, a vowel sequence would seem to lie outside the boundaries for recognition of a particular linguistic form. However, it is known that following presentation of a repeated syllable such as /ta/, the recognition boundaries corresponding to the previously presented $/ \mathrm{ta} / \mathrm{are}$ constricted. At the same time, the boundaries of templates responding to contrasting forms (e.g., /da/) are expanded so that they respond to tokens that would have been rejected previously. Thus, after repetitions of /ta/ are terminated, a stimulus ordinarily heard as $/ \mathrm{ta} /$ and having a voice-onset time near the normal $/ \mathrm{ta} /-/ \mathrm{da} /$ boundary may be heard as /da/ (Diehl, 1981; Miller \& Eimas, 1982). This shift in category boundaries is considerably greater when it is observed during rather than following repetition. Thus, during listening to a repeated utterance (an actual word or syllable), activation of an inappropriate template can occur after several seconds, resulting in "verbal transformation" into an illusory form in which any or all phonetic components of the utterance may be changed (Warren, 1968; Warren \& Myers, 1987). However, the verbal transformations heard during repetition of syllables and words are not subject to the spontaneous spectral splitting into simultaneous forms heard during repetition of vowel sequences.

In an attempt to understand why spectral splitting occurs, Warren et al. (1990) reasoned that although there is a tendency to match sequences of speech sounds to syllabic and lexical templates, this matching is difficult for a series of steady-state vowels. However, a match could be facilitated by extracting appropriate regions from the broadband stimulus and comparing them with spectrally abbreviated versions of linguistic templates. (As will be discussed shortly, there is evidence for such templates, since we can identify spoken words and sentences on the basis of spectrally limited regions.) The residue of the vowel sequence remaining after this extraction is occasionally perceived as an extraneous nonlinguistic sound occurring along with the illusory verbal form, but it is usually matched to another abbreviated verbal template and heard as a second form that differs in both phonetic components and timbre from the other. In a subsequent study, Chalikia and Warren (1991) successfully mapped the perceptual phonemes occurring in each of these forms to the recorded vowels occurring at the same time, by abruptly terminating the repeated sequence at predetermined positions and determining the last speech sound heard by the listener. It was found that the same phone could contribute to a different illusory speech sound for each of the forms. However, it was not possible on the basis of this earlier study to determine whether the same spectral regions of the phone contributed to both forms, or whether each form represented a different spectral region.
We designed Experiment 1 of the present study to determine whether it was possible to isolate separate spectral regions corresponding to each of the illusory forms. The use of bandpass filtering demonstrated that different spectral ranges were employed for each of the forms: one form usually corresponded to a band of frequencies below $1500 \mathrm{~Hz}$ and the other to a band of frequencies above $1500 \mathrm{~Hz}$. Interestingly, there is considerable evidence that a frequency of approximately $1500 \pm 300 \mathrm{~Hz}$ (depending on stimuli and procedures) divides speech into highpass and low-pass ranges of equal intelligibility (Beranek, 1947; Fletcher \& Galt, 1950; Hirsh, Reynolds, \& Joseph, 1954; Pollack \& Pickett, 1964; Studebaker, Pavlovic, \& Sherbecoe, 1987). Sentences provide higher intelligibility scores than do word lists, and each of two nonoverlapping spectral ranges can be quite intelligible when heard alone. Thus, when Bashford and Warren (1987) used Central Institute for the Deaf sentence lists, which were either low-pass filtered at $1100 \mathrm{~Hz}$ or high-pass filtered at $1700 \mathrm{~Hz}$ (their "baseline condition"), intelligibility scores of $98 \%$ were obtained in each condition.

Perceptual splitting of broadband phonemes does not usually occur. Vowels heard in isolation and vowels incorporated within normal utterances exhibit a spectral cohesion and are heard as single auditory images. We designed Experiment 2 to obtain an independent quantitative measure of the spectral cohesion remaining within vowel sequences by introducing an opposing force tending to divide the stimulus into spatially separate images. A contralateral induction paradigm was used, in which a broadband vowel sequence was presented to one ear, and a sound encompassing a frequency range corresponding to one of the verbal forms (as determined in Experiment 1) was presented to the opposite ear. The ears receiving each sound were reversed each $700 \mathrm{msec}$, and the minimum amplitude of the narrowband sound required to extract the corresponding illusory form from the lateralized broadband vowel sequence and move it to the medial plane was determined. This amplitude was taken as a measure of the force of contralateral induction that was necessary to overcome the cohesive force binding different spectral regions of the vowels into a common lateral position. It was found that delateralization of a form could be accomplished using either a bandpassed portion of the broadband vowel sequence or a bandpassed noise (the narrowband sound derived from the vowel sequence was more effective, producing delateralization at a lower amplitude than did the noise). When an extended statement of a single vowel or a real utterance was substituted for the vowel sequence, delateralization could not be accomplished at any level with either type of narrowband sound.

Illusions can reveal perceptual mechanisms that ordinarily remain hidden, and we suggest that the separate perceptual organization of high and low spectral components of vowel sequences may reflect an inappropriate operation of skills that normally enhance intelligibility. The ability to comprehend speech utilizing only a limited portion 
of its spectral range can be observed when extraneous sounds replace or mask some of the component frequencies but not others (Bashford \& Warren, 1987). In addition, the ability to use separate spectral regions for independent verbal organization may provide a means of checking perceptual accuracy when there is uncertainty and ambiguity concerning an utterance. However, when different spectral ranges of a broadband vowel sequence are processed independently, the resulting verbal organizations differ considerably both from the stimulus and from each other. Since these concurrent band-limited forms are quite different, the broadband spectral fusion exhibited by normal utterances is blocked, and listeners hear two simultaneous voices with distinguishable timbres, each saying something different.

\section{REFERENCES}

amano, S., Kashino, M., \& Warren, R. M. (1990). Perception of repeated vowel sequences. Proceedings of the Acoustical Society of Japan, 1, 307-308. (Abstract in Japanese)

Bartlett, F. C., \& MARK, H. A. (1922). A note on local fatigue in the auditory system. British Journal of Psychology, 13, 215-218.

BAShFord, J. A., JR., \& WARREN, R. M. (1987). Effects of spectral alternation on the intelligibility of words and sentences. Perception \& Psychophysics, 42, 431-438.

BeraneK, L. L. (1947). Design of speech communication systems. Proceedings of the Institute of Radio Engineers, 35, 880-890.

Butler, R. A., \& NAUnton, R. F. (1962). Some effects of unilateral auditory masking upon the localization of sound in space. Journal of the Acoustical Society of America, 34, 1100-1107.

Butler, R. A., \& Naunton, R. F. (1964). Role of stimulus frequency and duration in the phenomenon of localization shifts. Journal of the Acoustical Society of America, 36, 917-922.

Chalikia, M. H., \& Warren, R. M. (1991). Phonemic transformations: Mapping the illusory organization of steady-state vowel sequences. Language \& Speech, 34, 109-143.

Cherry, E. C., \& TAYLOR, W. K. (1954). Some further experiments upon the recognition of speech, with one and with two ears. Joumal of the Acoustical Society of America, 26, 554-559.

Cole, R. A., \& ScotT, B. (1973). Perception of temporal order in speech: The role of vowel transitions. Canadian Journal of Psychology, 27, 441-449.

Culunan, W. L., Erdos, E., Schaefer, R., \& Tekiel, M. E. (1977). Perception of temporal order of vowels and consonant-vowel syllables. Journal of Speech \& Hearing Research, 20, 742-751.

DEuTsCH, D. (1979). Binaural integration of melodic patterns. Perception \& Psychophysics, 25, 399-405.

DiEHL, R. L. (1981). Feature detectors for speech: A critical reappraisal. Psychological Bulletin, 89, 1-18.

Dorman, M. F., Cutting, J. E., \& Raphael, L. J. (1975). Perception of temporal order in vowel sequences with and without formant transitions. Joumal of Experimental Psychology: Human Perception \& Performance, 11, 209-220.

Efron, R. (1963). Temporal perception, aphasia and déjà vu. Brain, 86, 403-424.

EgAN, J. P. (1948). The effect of noise in one ear upon the loudness of speech in the other. Journal of the Acoustical Society of America, 20, 58-62.

FLETChER, H., \& GALT, R. H. (1950). The perception of speech and its relation to telephony. Journal of the Acoustical Society of America, 22, 89-151.

Flugel, J. C. (1920). On local fatigue in the auditory system. British Journal of Psychology, 11, 105-134.

FoulKe, E., \& STICHT, T. G. (1969). Review of research on the intelligibility and comprehension of accelerated speech. Psychological Bulletin, 72, 50-62.
Hirsh, I. J., Reynolds, E. G., \& JosePh, M. (1954). Intelligibility of different speech materials. Journal of the Acoustical Society of America, 26, 530-538.

Miller, J. L., \& Eimas, P. D. (1982). Feature detectors and speech perception: A critical evaluation. In D. G. Albrecht (Ed.), Recognition of pattern and form (pp. 111-145). New York: Springer-Verlag.

Pollack, I., \& PicketT, J. M. (1964). Frequency importance function for isolated words and for conversation of female talkers. Language \& Speech, 7, 71-75.

RIENER, K. R., \& WARREN, R. M. (1990). Verbal organization of vowel sequences: Effects of repetition rate and stimulus complexity. Journal of the Acoustical Society of America, 88, S55. (Abstract)

Schubert, E. D., \& Parker, C. D. (1956). Addition to Cherry's findings on switching speech between the two ears. Journal of the Acoustical Society of America, 27, 792-794.

STEIGER, H., \& BREGMAN, A. S. (1982). Negating the effects of binaural cues: Competition between auditory streaming and contralateral induction. Journal of Experimental Psychology: Human Perception \& Performance, 8, 602-613.

Studebaker, G. A., Pavlovic, C. V., \& Sherbecoe, R. L. (1987). A frequency importance function for continuous discourse. Journal of the Acoustical Society of America, 81, 1130-1138.

Thomas, I. B., Cetti, R. P., \& Chase, P. W. (1971). Effect of silent intervals on the perception of temporal order for vowels. Journal of the Acoustical Society of America, 49, 84. (Abstract)

Thomas, I. B., Hill, P. B., Carroll, F. S., \& Garcia, B. (1970). Temporal order in the perception of vowels. Journal of the Acoustical Society of America, 48, 1010-1013.

Thurlow, W. R., \& Elfner, L. F. (1959). Continuity effects with alternately sounding tones. Journal of the Acoustical Society of America, 31, 1337-1339.

WARREN, R. M. (1968). Verbal transformation effect and auditory perceptual mechanisms. Psychological Bulletin, 70, 261-270.

W ARREN, R. M. (1971). Identification times for phonemic components of graded complexity and for spelling of speech. Perception \& Psychophysics, 9 (4), 345-349.

WARREN, R. M. (1993). Perception of acoustic sequences: Global integration versus temporal resolution. In S. McAdams and E. Bigand (Eds.), Thinking in sound: The cognitive psychology of human audition (pp. 37-68). Oxford: Oxford University Press.

WARREN, R. M., \& BASHFORD, J. A. [JR.] (1976). Auditory contralateral induction: An early stage in binaural processing. Perception \& Psychophysics, 20, 380-386.

WARREN, R. M., BASHFord, J. A., JR., \& GARDNer, D. A. (1990). Tweaking the lexicon: Organization of vowel sequences into words. Perception \& Psychophysics, 47, 423-432.

WARREN, R. M., \& MYers, M. D. (1987). Effects of listening to repeated syllables: Category boundary shifts versus verbal transformations. Journal of Phonetics, 15, 169-181.

Warren, R. M., Obusek, C. J., Farmer, R. M., \& Warren, R. P. (1969). Auditory sequence: Confusion of patterns other than speech or music. Science, 164, 586-587.

Warren, R. M., \& Sherman, G. L. (1974). Phonemic restorations based on subsequent context. Perception \& Psychophysics, 16, 150-156.

Warren, R. M., \& Warren, R. P. (1970, December). Auditory illusions and confusions. Scientific American, pp. 30-36.

\section{NOTES}

1. When contralateral induction of a monaural signal is produced by a noise delivered to the opposite ear, a lateralized residue of noise is heard. However, in this study, when the inducing sound was filtered speech, there was no such residue. The presence or absence of a residue appears to reflect the subtractive nature of contralateral induction. When a monaural broadband speech signal is paired with a contralateral narrowband noise, components matched in spectrum and amplitude are subtracted from each side and moved to the center; the unmatched components of the noise and the speech remain lateralized. However, if the inducing sound is bandpass speech that has the same spectral amplitude profile at each moment as the corresponding frequency region of the 
contralateral broadband speech, then only the broadband speech has a lateralized unmatched residue and there is no residue left on the side of the narrowband speech

2. In order to test whether the observations made concerning the spectral cohesion of steady-state vowels apply to sounds that are not derived from speech, a $100-\mathrm{Hz}$ pulse train was substituted for the extended statements of steady-state vowels - this complex tone approximates the acoustic characteristics of glottal pulses before formant shaping by the resonant cavities of the vocal tract. As with the steady-state vowels, contralateral induction did not occur with any level of an inducing sound consisting of either a narrowband portion of the signal or a narrowband noise. As a further test of the validity of using contralateral induction as a measure of spectral cohesion, we reasoned that cohesion should be absent for the stochastically independent spectral components of a monaural broadband noise. This prediction was confirmed when a monaural narrowband noise was paired with a contralateral broadband noise, with the sounds switching sides every $700 \mathrm{msec}$. The narrowband inducing noise could be produced either by on-line filtering of the contralateral broadband noise signal or by filtering noise from an independent source-in both cases, there was no evidence of spectral cohesion, and delateralization of the common spectral components occurred when the narrowband noise was within $1 \mathrm{~dB}$ of the level of the same frequency region of the contralateral broadband noise.

(Manuscript received November 15, 1991; revision accepted for publication July 9, 1993.) 